\title{
Agrobiodiversidad En La Comunidad Joyagshi, Chunchi-Ecuador
}

\author{
Edmundo Danilo Guilcapi Pacheco \\ Christiam Paúl Aguirre Merino \\ Danny Daniel Castillo Vizuete \\ Leidy Estefanía Álvarez Molina \\ Gabriela Natalí Fonseca Romero
}

Investigadores, Escuela Superior Politécnica de Chimborazo

Doi: 10.19044/esj.2017.v13n33p53 URL:http://dx.doi.org/10.19044/esj.2017.v13n33p53

\begin{abstract}
Ecuador is one of the most biologically rich countries in the world. It is considered as a mega diverse country, meaning that it has the most varied life forms expressed in its flora, fauna, microorganisms, genetic diversity and a variety of ecosystems, which are formed thanks to the particular geographical conditions of location, relief and climate. According to the territorial location, Llagos is the last parish of the province of Chimborazo, it belongs to the Chunchi canton and is made up of 11 communities, is based largely on an irregular terrain of extreme slope in certain sectors, is rich in biodiversity of Flora and fauna. Joyagshi is one of the communities of the parish, which is made up of plains, plateaus and few hills. Thus, from the identification of the flora inventory of the Joyagshi community and in addition to the morphological and taxonomic identification of the plants, a study of agrobiodiversity in the community was elaborated. In conclusion, 44 species of flora of the community of Joyagshi were inventoried in a total of 170 hectares, which are destined for diverse purposes like: pastures, fodder, crops, and natural forests.
\end{abstract}

Keywords: Agrobiodiversity, biodiversity, flora, morphology, taxonomy, Joyagshi, Llagos, Chunchi, Ecuador

\section{Resumen}

Ecuador es uno de los países biológicamente más ricos del planeta, es considerado como un país mega diverso, ésto significa, que cuenta con las más variadas formas de vida expresadas en su flora, fauna, microorganismos, en su diversidad genética y en una variedad de ecosistemas, que se forman gracias a las particulares condiciones geográficas de ubicación, relieve y 
clima. De acuerdo a la ubicación territorial, Llagos es la última parroquia de la provincia de Chimborazo, pertenece al cantón Chunchi y está conformada por 11 comunidades, se asienta en gran parte sobre un terreno irregular de extrema pendiente en ciertos sectores, es rica en biodiversidad de flora y fauna. Joyagshi es una de las comunidades de la parroquia, la misma que está conformada por llanuras, mesetas y pocas colinas. Es así que, a partir de la identificación del inventario de flora de la comunidad Joyagshi y además de la identificación morfológica y taxonómica de las plantas se elaboró un estudio de agrobiodiversidad en la comunidad. En conclusión se inventariaron 44 especies de flora de la comunidad de Joyagshi en un total de 170 hectáreas, las cuales son destinadas para diversos fines como: pastos, forrajes, cultivos y bosques naturales.

Palabras claves: Agrobiodiversidad, biodiversidad, flora, morfología, taxonomía, Joyagshi, Llagos, Chunchi, Ecuador

\section{Introducción}

Según (Hernández, 2017) la pobreza es un estado de debilidad, de dependencia, de subordinación o humillación, respecto a la privación de medios para conseguir la subsistencia, para una existencia humanamente digna; medios de todo tipo: económicos, sociales, de poder o saber, de salud, de honra, etc. Adicionalmente, se comprende la pobreza como una capacidad disminuida que afecta al individuo en sus relaciones sociales, culturales, políticas, laborales, tal combinación dinámica puede llevar a la exclusión e implica una vulnerabilidad ante problemas físicos y psicológicos (Landa y Landa, 2017). Para el (PNUD, 2001) la pobreza desde la perspectiva del desarrollo humano, significa que se priven las oportunidades y las opciones más fundamentales del desarrollo humano: vivir una larga vida, sana y creativa, disfrutar de un nivel de vida decente, libertad, respeto por sí mismo, entre otras. Según (Vite, 2007) la pobreza se vincula con la precariedad del trabajo, manifestada como una insuficiencia del salario para acceder a bienes y servicios, generados por el mercado y el Estado.

Al hablar de pobreza rural, (Echeverría, 2011) manifiesta que la misma es más aguda que la urbana y parece más difícil de superarla. Por su parte (Klein, 2012) sostiene que la pobreza rural se genera por la informalidad y la precariedad en el empleo. La realidad de las familias rurales no ha sido de interés, y en general poco se cuestiona los efectos del cambio en el patrón global de acumulación con la profundización de las desigualdades campo-ciudad, el deterioro de la producción sostenible en el campo y el surgimiento de formas de familia no tradicionales derivadas de procesos de desintegración con arraigo socioeconómico (Mendoza, 2017). Los humildes campesinos pobres están en desventaja por varias razones que 
se interrelacionan: la mayoría viven en áreas muy remotas; suelen tener mala salud y ser analfabetos; tienen familias numerosas, ocupan puestos de trabajo inseguros y relativamente poco productivos, y muchos son explotados y discriminados por su condición de mujeres o de miembros de minorías étnicas; y todo eso como el resultado de las malas políticas sociales (Alvarado, 2007).

El rol de la agrobiodiversidad en los agroecosistemas ha sido revalorizado en los últimos años por los servicios ecológicos que brinda, tales como el ciclado de nutrientes, la regulación biótica, el mantenimiento del ciclo hidrológico, la polinización, entre otros (Stupino et al., 2014). La función económica de la agrobiodiversidad está direccionada al consumo y a la comercialización (Ríos y Camacho, 2016). Según (Castro et al., 2005) el mantenimiento de la agrobiodiversidad contribuye a diversificar productos y oportunidades de ingreso para los productores, a reducir la dependencia de materiales genéticos foráneos, a conservar la estructura de los ecosistemas haciéndolos más estables y sostenibles, y a aumentar el empoderamiento y la participación de granjeros y personas de la comunidad. La agrobiodiversidad incluye todos los componentes de la diversidad biológica pertinentes a la alimentación, la agricultura y el ecosistema agrícola, así como un fuerte componente sociocultural, puesto que la diversidad biológica agrícola está en gran parte determinada por actividades humanas, saberes de los productores y prácticas de gestión (Sarandón, 2009).

Bajo este contexto el presente artículo tiene como objetivo identificar el inventario de flora de la comunidad Joyagshi, además de realizar la identificación morfológica y taxonómica de las plantas con la finalidad de elaborar el estudio de agrobiodiversidad actual de la comunidad Joyagshi.

\section{Metodología}

\section{Localización}

La investigación se llevó a cabo en el área arqueológica del monte Puñay, Comunidad Joyagshi, Parroquia Llagos, Cantón Chunchi, Provincia de Chimborazo. Es una zona de fácil accesibilidad, posee una temperatura promedio anual es de $12-20^{\circ} \mathrm{C}$, mientras que la precipitación promedio anual es de $700 \mathrm{~mm}$, la humedad relativa alcanza el $88 \%$. 


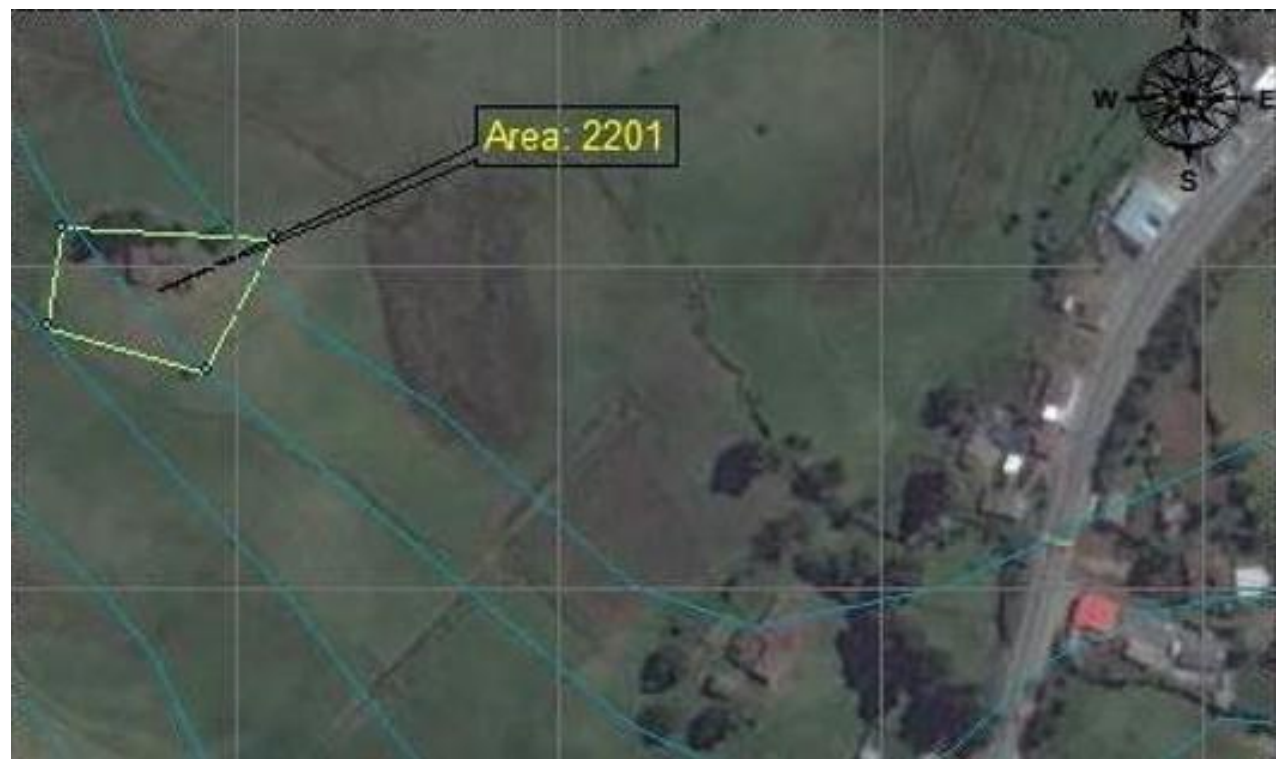

Gráfico 1. Mapa de localización de la comunidad Joyagshi

\section{Ubicación Geográfica}

Coordenadas proyectadas UTM Zona $17 \mathrm{~S}$

Datum WGS 84

$\mathrm{X}: 726012 \mathrm{E}$

Y: $9737960 \mathrm{~N}$

\section{Realización del inventario de flora de la comunidad Joyagshi.}

Mediante un análisis preliminar del mapa del lugar, el mismo que se obtuvo a través del GPS se determinó: área total, curvas de nivel; teniendo así un área de estudio de $7440 \mathrm{~m}$. Posterior a esto, se hizo un reconocimiento del área determinando que es un terreno extenso, de fácil accesibilidad y con abundantes pastos, plantas nativas y muy pocos cultivos. Para realizar el inventario se hicieron 7 transectos lineales de 20 x $5 \mathrm{~m}$. Mediante salidas de campo se inventarió las especies de la comunidad Joyagshi.

\section{Identificación morfológica y taxonómica}

A través de revisión documental se realizó el estudio morfológico de las plantas recolectadas, mismas que presentan características como: forma, tamaño, espesor, alto, ancho, entre otros. Así también mediante el mismo método se realizó el estudio taxonómico en donde se logró determinar el orden, género, nombre científico, familias, etc. 


\section{Análisis de datos}

Con la información obtenida insitu se realizó un análisis de biodiversidad mediante el programa PAST obteniendo los índices de Shannon, Margalef, Simpsom, con el fin de obtener un registro y datos muy importantes para valorar las especies vegetales de la zona.

\section{Resultados}

Inventario de especies

Cuadro 1 Matriz resumen de la flora de la comunidad de Joyagshi

Elaboración: Propia

\begin{tabular}{|c|c|c|c|c|c|c|}
\hline \multirow{2}{*}{$\mathbf{N}^{\mathbf{o}}$} & \multirow{2}{*}{ Nombre vulgar } & \multicolumn{3}{|c|}{ Taxonomía } & \multirow{2}{*}{ Morfología } & \multirow{2}{*}{ Usos } \\
\hline & & Nombre científico & Familia & Tipo de vegetación & & \\
\hline \multirow{3}{*}{1} & & \multirow{3}{*}{$\begin{array}{l}\text { Delostoma } \\
\text { integrifolium }\end{array}$} & \multirow{3}{*}{ Bignoniaceae } & \multirow{3}{*}{ Arbórea } & Hojas: Hojas simples con 3 venas desde la base. & \multirow{3}{*}{$\begin{array}{l}\text { Ornamental, protección del } \\
\text { suelo, reforestación }\end{array}$} \\
\hline & & & & & $\begin{array}{l}\text { Flores: Racimos o panículas con } 2-3 \text { flores. } \\
\text { Flores con cáliz grande, cupuliforme, usualmente } \\
\text { doble; corola tubular a campanulada, conspicua, } \\
\text { magenta a blanca. Cápsula elíptica, comprimida } \\
\text { paralelamente al septo, valvas desiguales; sus } \\
\text { flores son muy llamativas de un color morado. }\end{array}$ & \\
\hline & & & & & $\begin{array}{l}\text { Semillas: Delgadas con una ala hialina, } \\
\text { membranácea. }\end{array}$ & \\
\hline \multirow{4}{*}{2} & & \multirow{4}{*}{$\begin{array}{l}\text { Oreopanax } \\
\text { ecuadorensis }\end{array}$} & \multirow{4}{*}{ Araliaceae } & \multirow{4}{*}{ Arbórea } & $\begin{array}{c}\text { Hojas: Hojas enteras, palmatilobadas o } \\
\text { palmaticompuestas; pecioladas, no liguladas. }\end{array}$ & \multirow{4}{*}{$\begin{array}{l}\text { Medicinal, reforestación, } \\
\text { protección del suelo }\end{array}$} \\
\hline & & & & & $\begin{array}{c}\text { Flores: Inflorescencia frecuentemente grande, } \\
\text { paniculada o simple-racemosa; flores sésiles, } \\
\text { bracteoladas; pétalos valvados; flores } \\
\text { hermafroditas. }\end{array}$ & \\
\hline & & & & & Fruto: Globoso. & \\
\hline & & & & & $\begin{array}{l}\text { Semillas: En igual o menor número que lóculos, } \\
\text { endosperma raramente liso. }\end{array}$ & \\
\hline 3 & Pucañahui & $\begin{array}{l}\text { Sauravia peruviana } \\
\text { Buscal }\end{array}$ & Actinidiaceae & Arbórea & $\begin{array}{c}\text { Tallo: Tiene una corteza leñosa de color marrón } \\
\text { claro o gris oscuro. }\end{array}$ & $\begin{array}{l}\text { Medicinal, ornamental, } \\
\text { reforestación }\end{array}$ \\
\hline
\end{tabular}




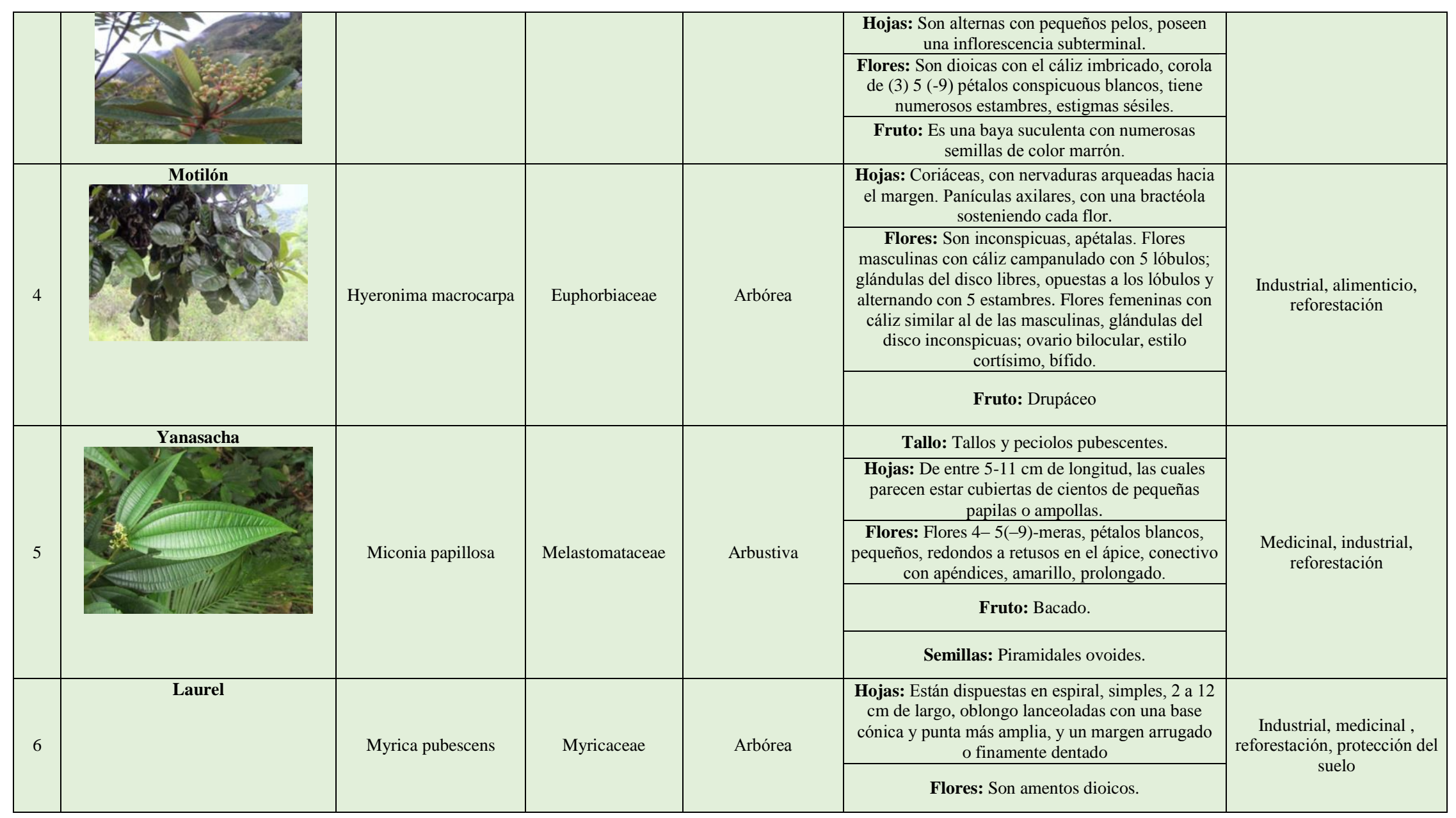




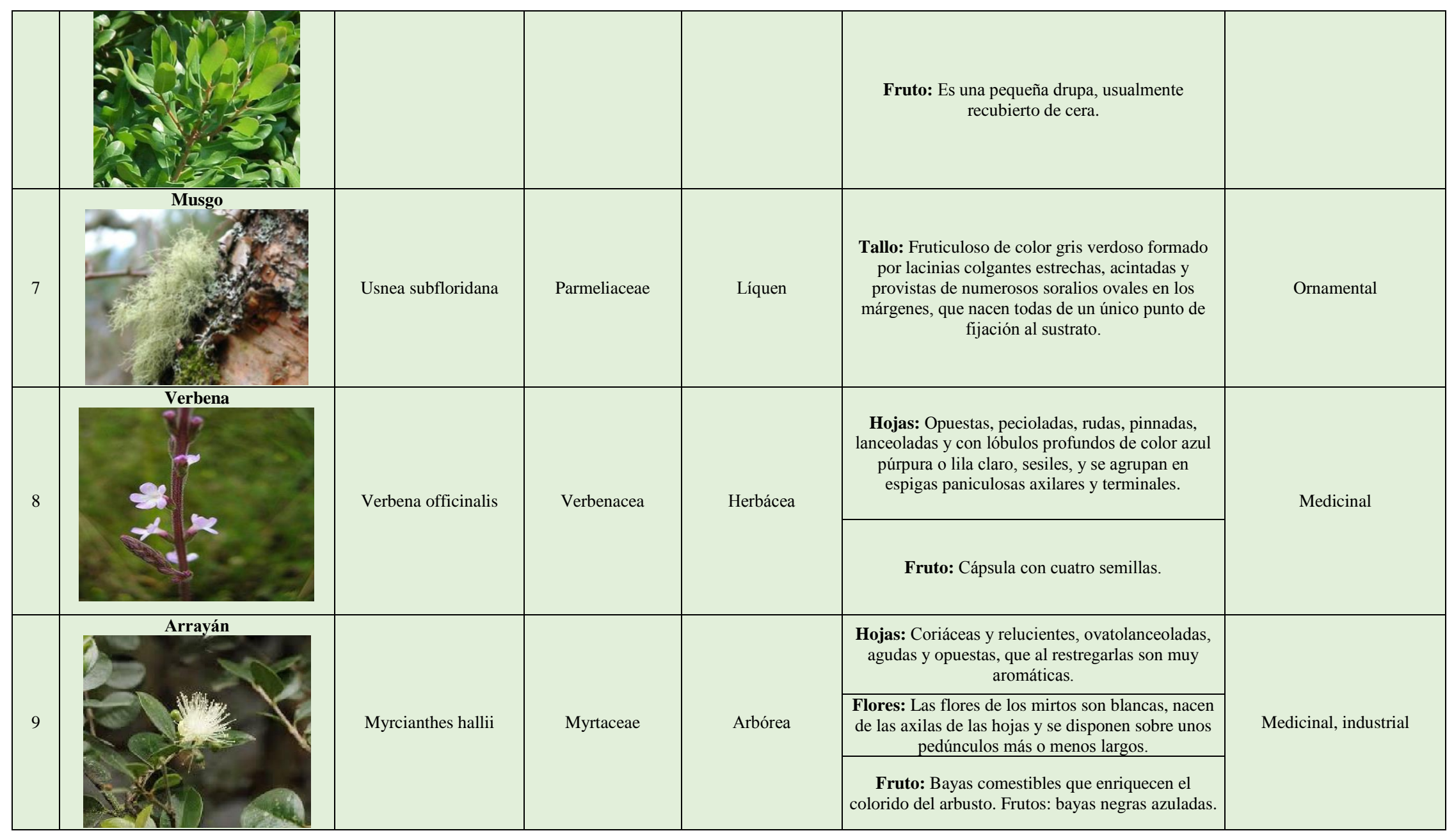




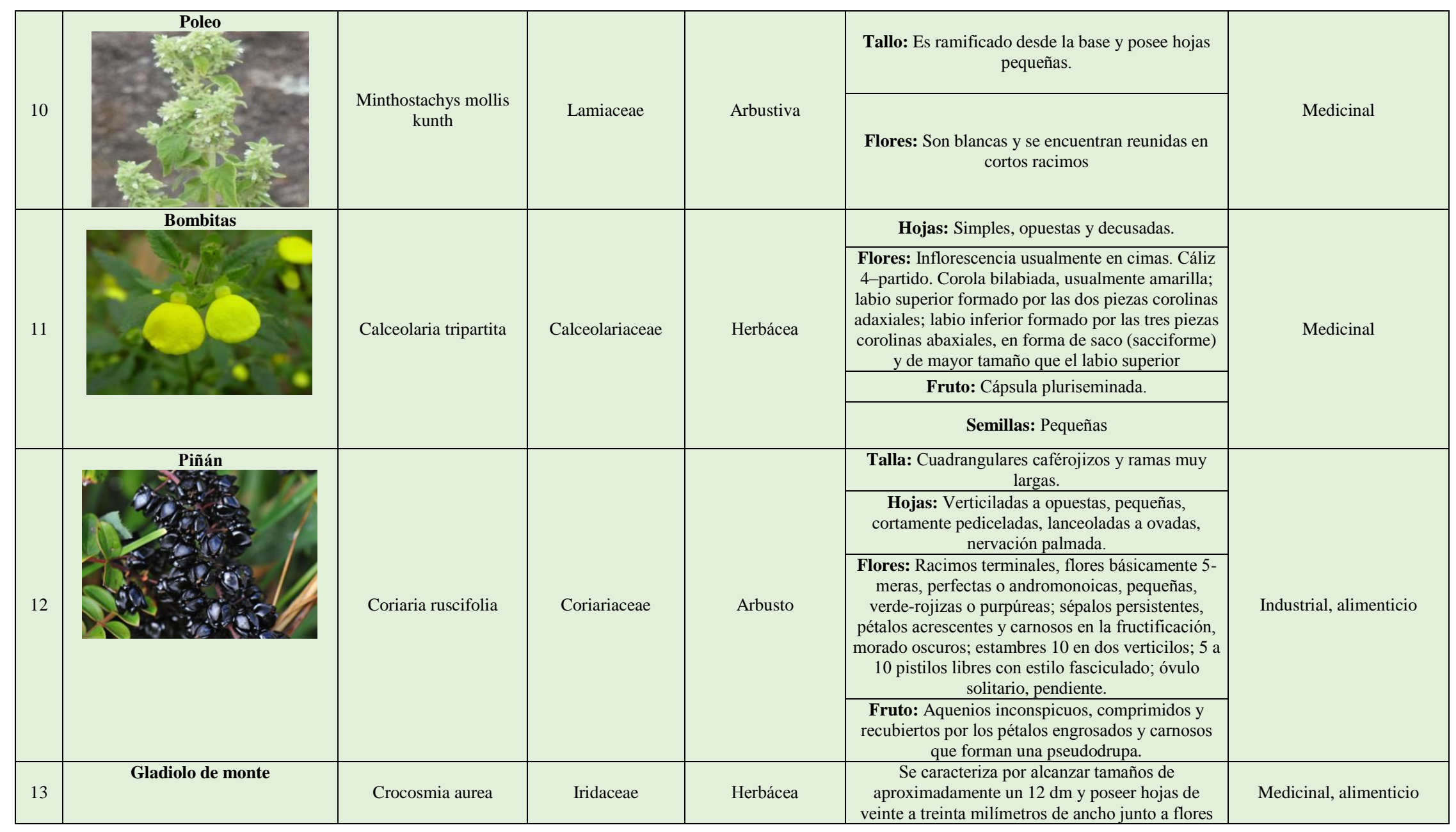




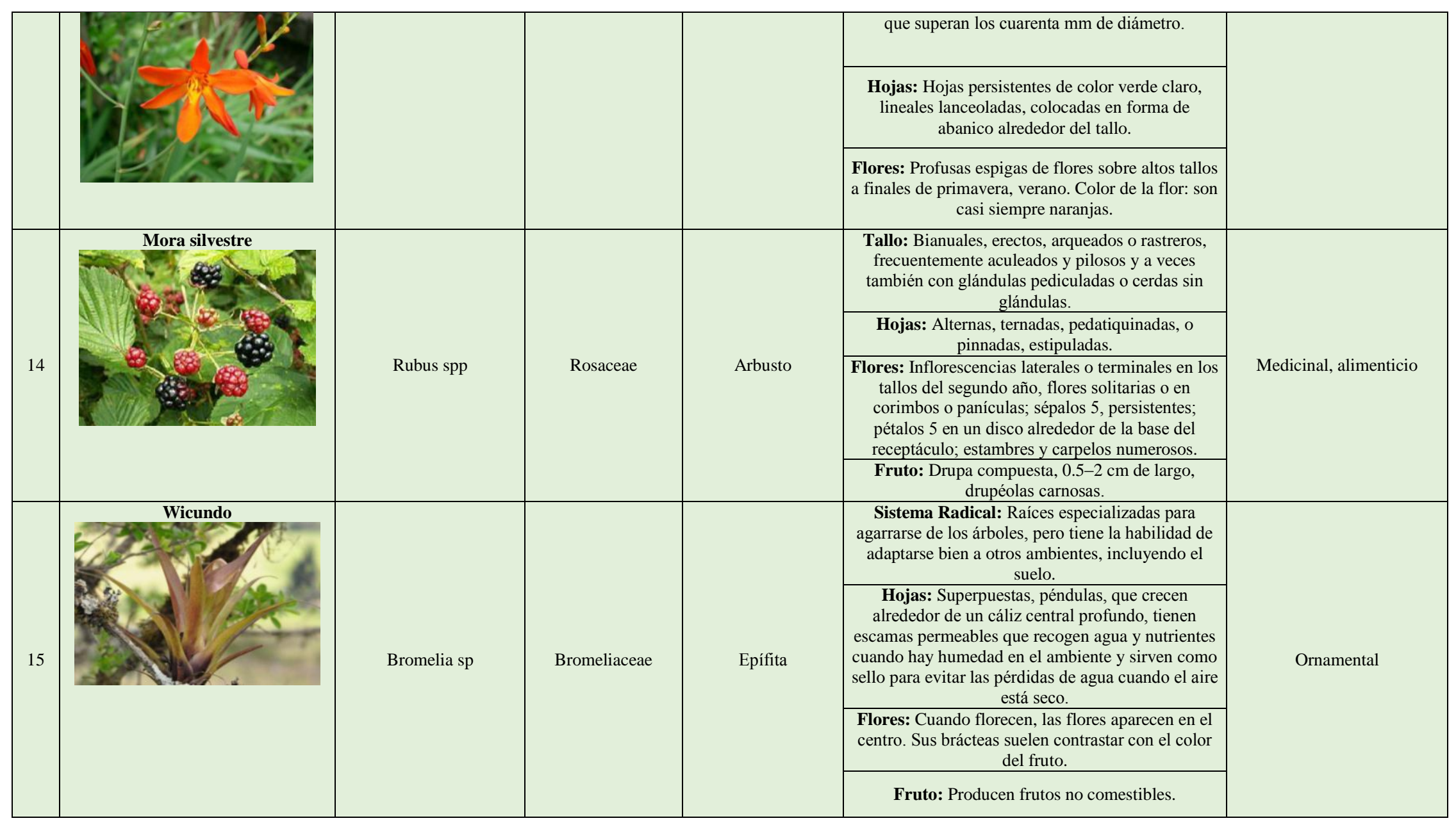




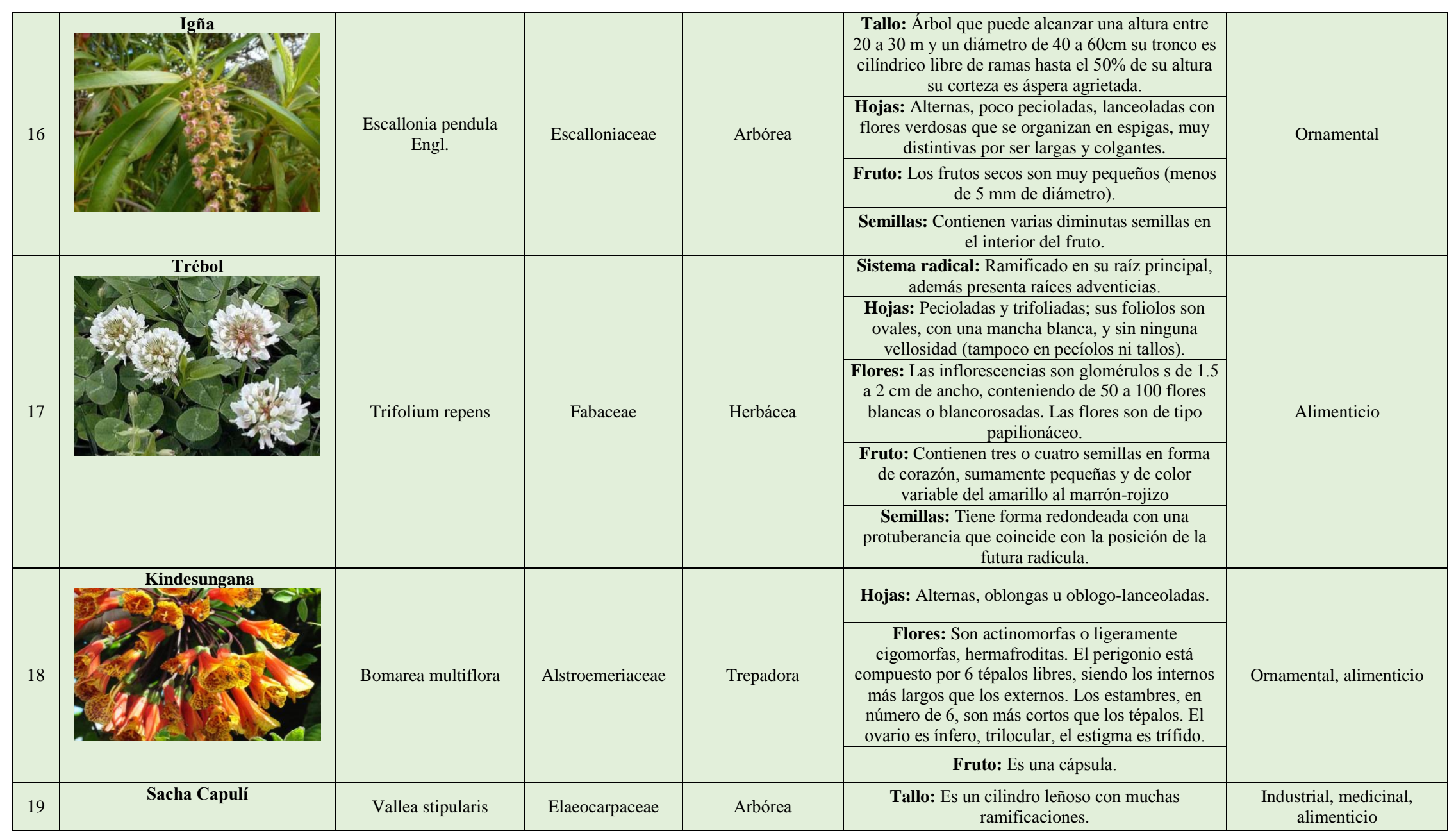




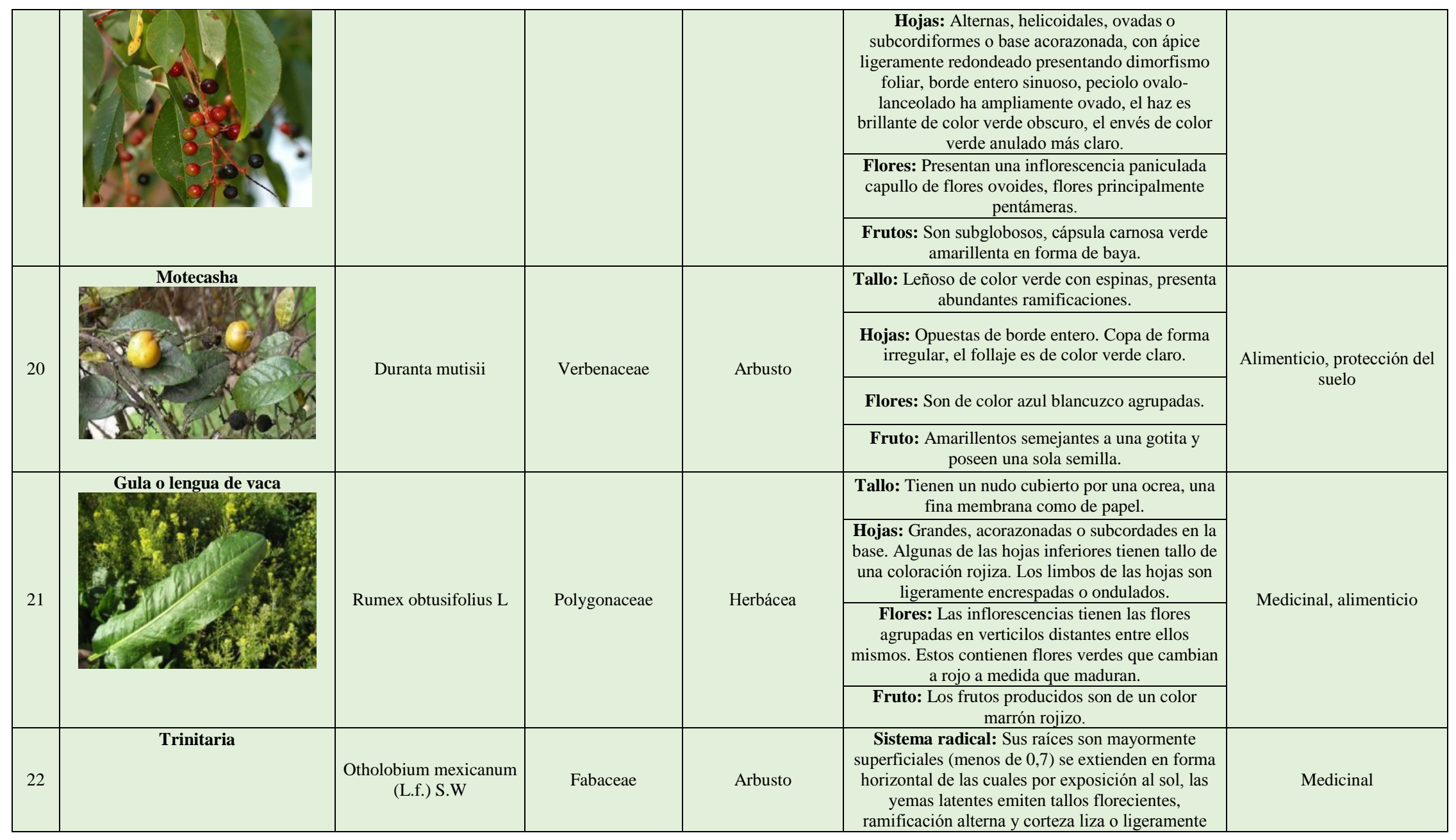




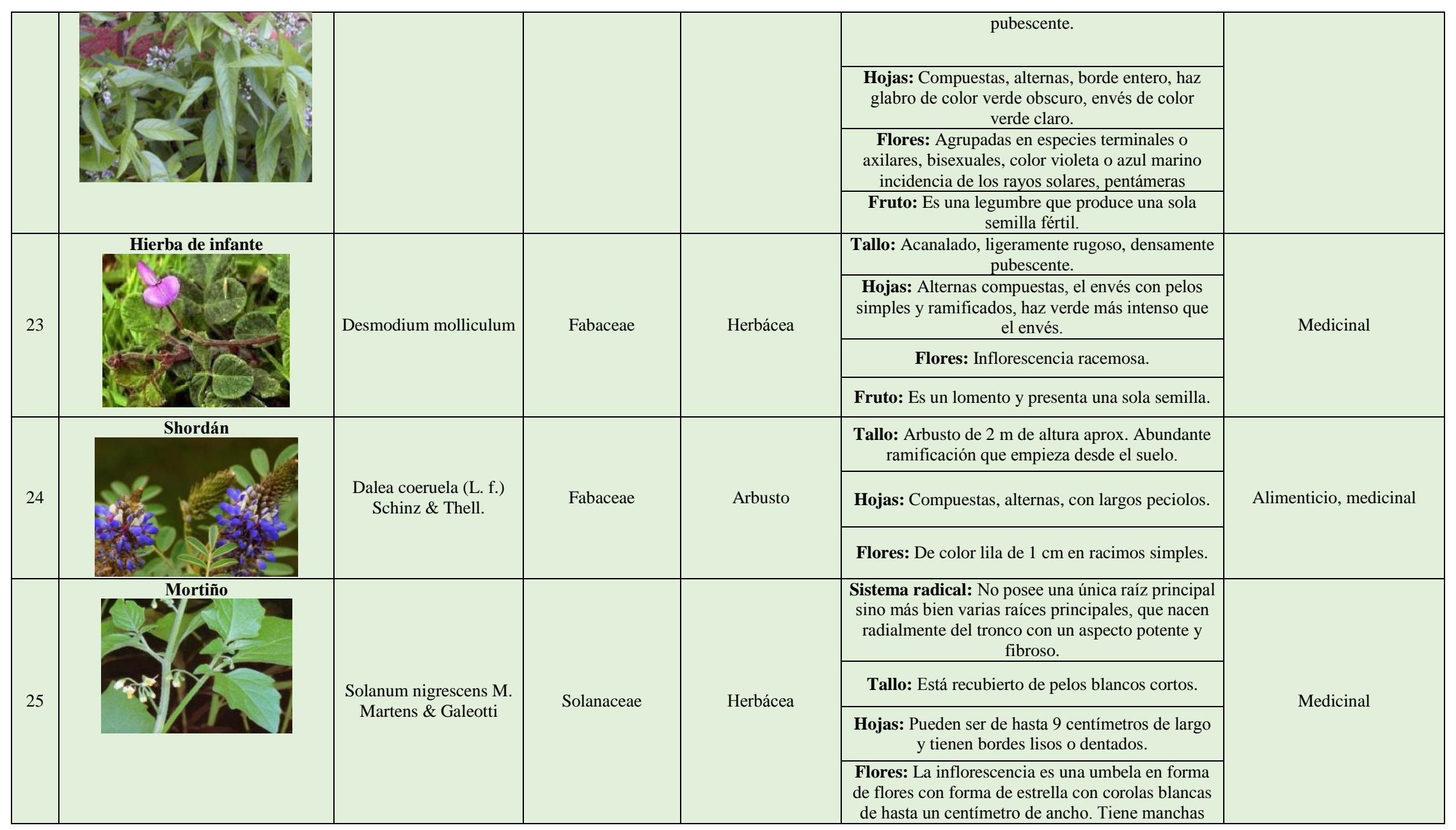




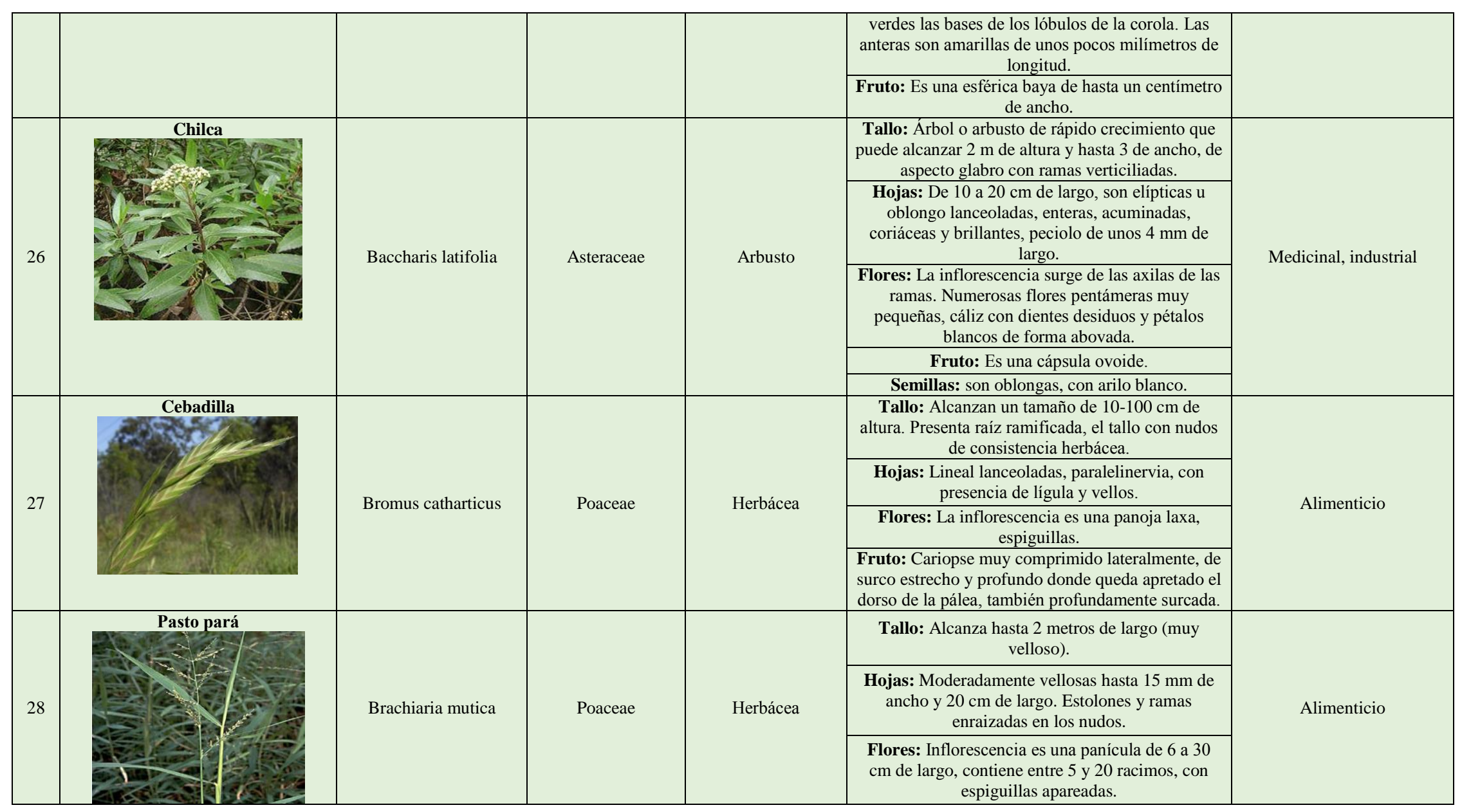




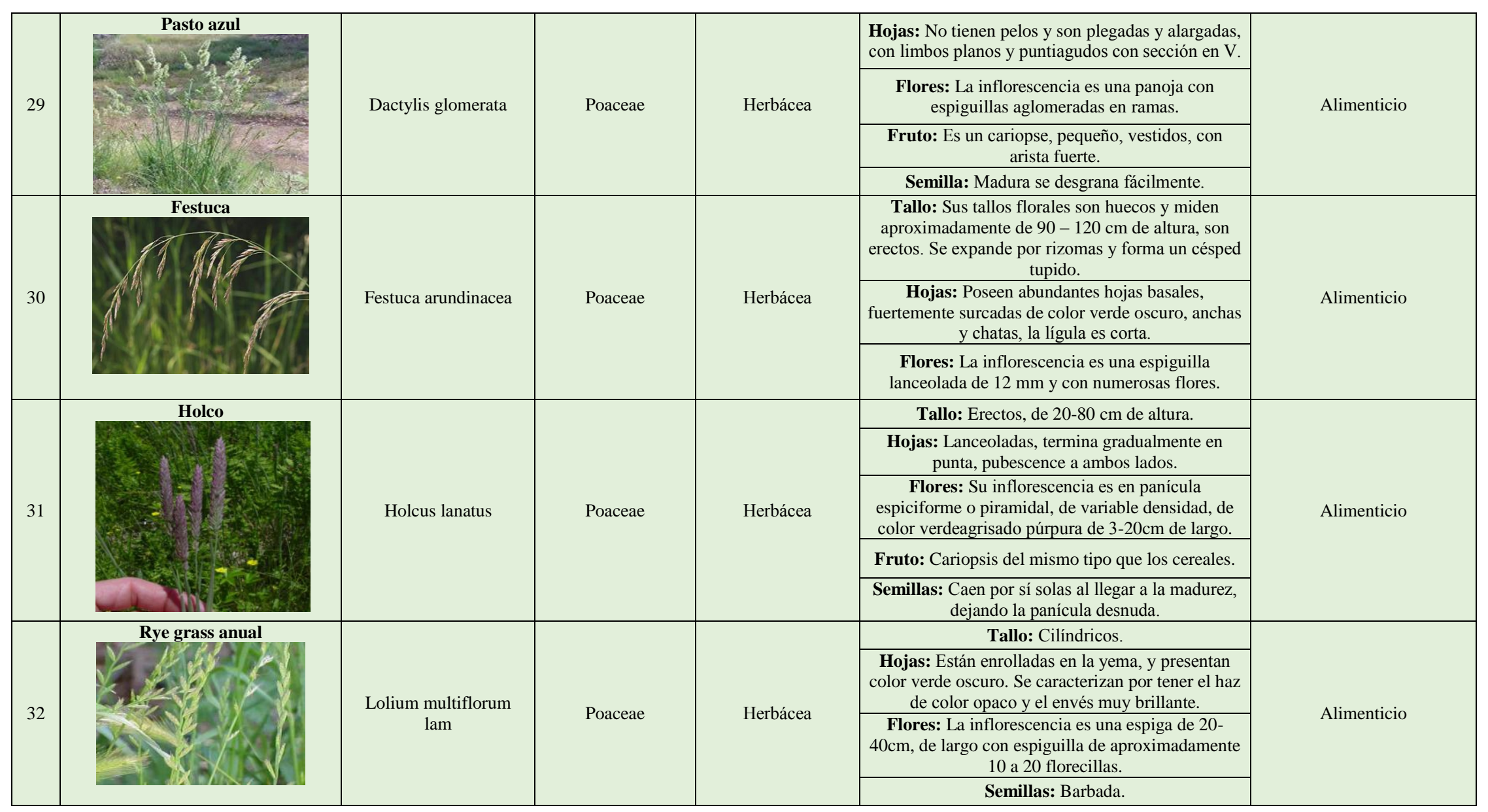




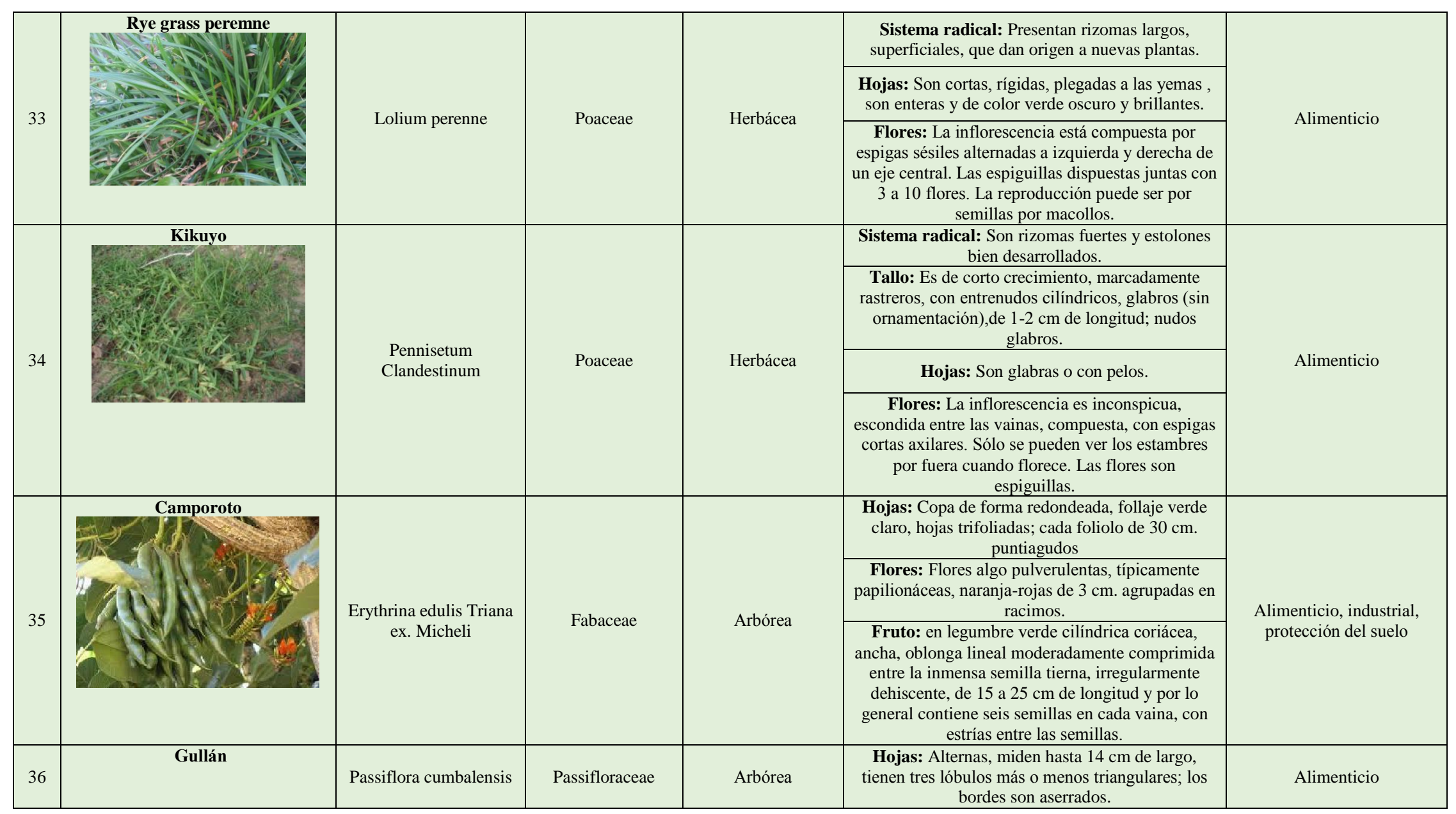




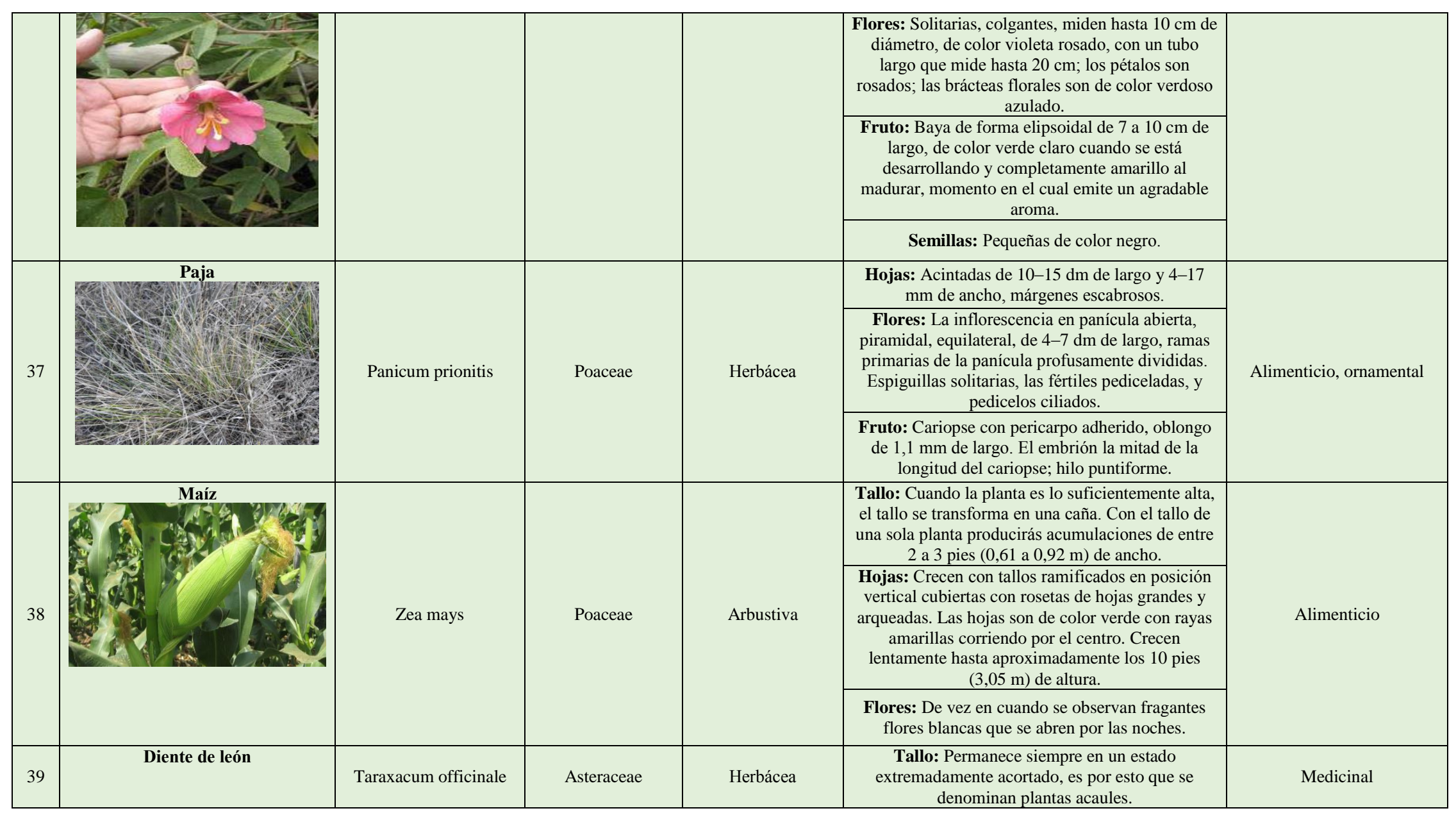




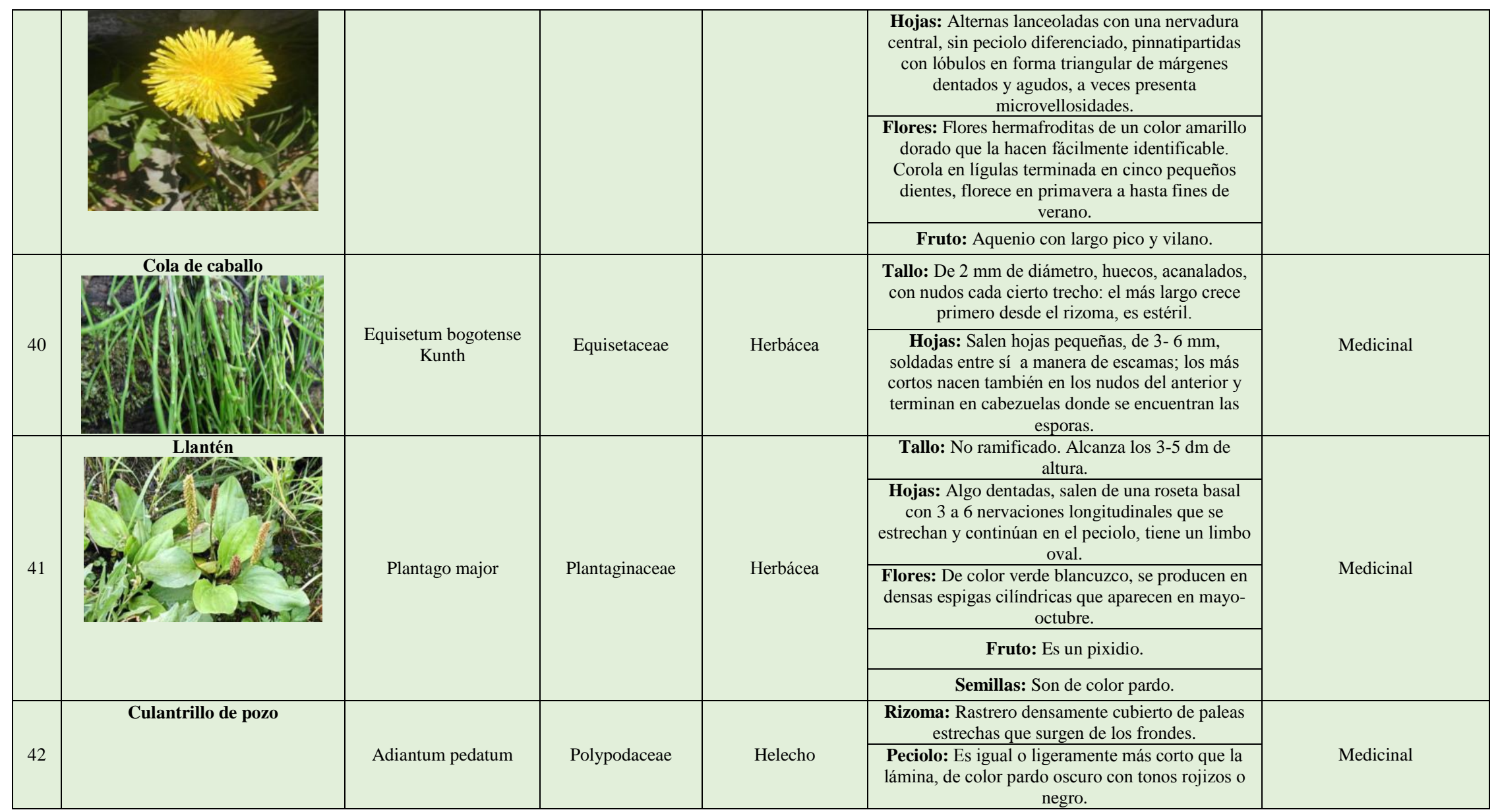




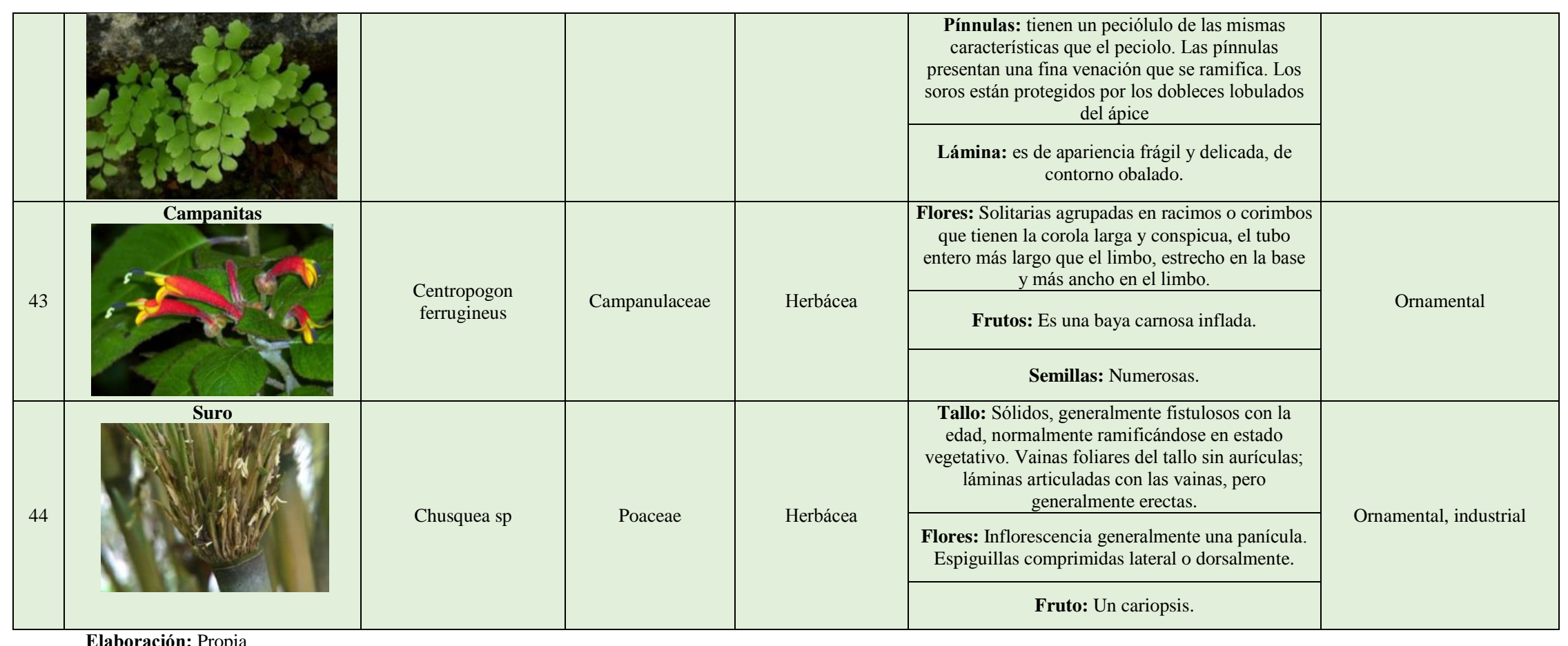

Elaboración: Propia 


\section{Utilización del suelo de la comunidad Joyagshi}

Cuadro 2. Utilización del suelo de la comunidad Joyagshi

\begin{tabular}{|c|c|c|}
\hline Utilización & Hectáreas & $\%$ \\
\hline Pastos y forrajes & 120 & $71 \%$ \\
\hline Remanente de bosques naturales & 25 & $15 \%$ \\
\hline Población & 15 & $9 \%$ \\
\hline Cultivos & 10 & $6 \%$ \\
\hline TOTAL & 170 & $100 \%$ \\
\hline
\end{tabular}

Elaboración: Propia

\section{Análisis de biodiversidad}

Cuadro 3. Análisis de biodiversidad

\begin{tabular}{|c|c|}
\hline Simpson_1-D & 0,9584 \\
\hline Shannon_H & 3,282 \\
\hline Margalef & 4,356 \\
\hline
\end{tabular}

Elaboración: Propia

Al determinar la diversidad según el índice de Margalef, se obtuvo como resultado un rango de 4,3; los valores inferiores a 2,0 son considerados zonas de baja biodiversidad y valores superiores a 5,0 son considerados como indicativos de alta biodiversidad, determinando que Joyagshi posee una diversidad media. De acuerdo al índice de Simpson se obtuvo un valor de 0,9584; los valores referenciales nos indican que mientras más aumente el valor a 1, la diversidad disminuye, definiendo por lo tanto que Joyagshi es un territorio con baja diversidad. Así también, mediante el índice de Shannon tenemos un resultado de 3,2 en la mayoría de los ecosistemas naturales varía entre 1 y 5, cuanto mayor sea este valor habrá una mayor diversidad en la zona. Valores por encima de 3 son interpretados como "diversos", en este caso se considera que Joyagshi es una zona media en cuanto a vegetación.

\section{Conclusiones}

- Se han inventariado 44 especies de flora de la comunidad de Joyagshi de las cuales se realizó la identificación morfológica y taxonómica, obteniendo el estudio de agrobiodiversidad de la comunidad.

- La comunidad Joyagshi cuenta con 170 hectáreas de las cuales el 70\% de las mismas son destinadas para pastos y forrajes, el $15 \%$ pertenece a remanente de bosques naturales, un $9 \%$ corresponde a la población y el $6 \%$ restante del suelo se lo utiliza para cultivos, por lo que se concluye que Joyagshi es una zona altamente ganadera. 
- A través del análisis de biodiversidad se obtuvo los índices de Margalef, Simpson y Shannon y se determinó que la comunidad de Joyagshi es una zona con una diversidad media en cuanto a vegetación.

\section{References:}

1. Alvarado J. (2007): "El sector agrícola en el combate de la pobreza rural". http://www.redalyc.org/articulo.oa?id $=72920534012$

2. Castro G., Lozano A., Fernández G., Rodríguez D. (2005): "Agrobiodiversidad y Pobreza" http://www.redalyc.org/articulo.oa?id=49520714

3. Echeverría R. (2011): "Opciones para reducir la pobreza rural en América Latina y el Caribe". Revista CEPAL 70.

4. Hernández A. (2017): "Reflexionando sobre la comprensión de la pobreza y la desigualdad así como sus implicaciones en el contexto actual".

5. https://www.researchgate.net/publication/317773731_Reflexionando _sobre_la_comprension_de_la_pobreza_y_la_desigualdad_asi_como _sus_implicaciones_en_el_contexto_actual?ev=srch_pub

6. Klein Emilio (2012). "Políticas de mercado de trabajo y pobreza rural en América Latina". Food and Agriculture Organization of the United Nations - FAO. Viale delle Terme di Caracalla, 00153 Roma (Italia). http://www.fao.org/3/a-i2651s.pdf. 9

7. Landa M., Landa A. (2017): "Determinantes de la pobreza subjetiva en habitantes del Municipio de Tatumbla, Honduras".

8. https://www.researchgate.net/publication/318100835_Determinantes _de_la_pobreza_subjetiva_en_habitantes_del_Municipio_de_Tatumb la_Honduras?ev=srch_pub

9. Mendoza A. (2017): "Pobreza rural y neoliberalismo: necesidades de las familias rurales en el municipio de Ciudad Barrios, Departamento de

San

Miguel". https://www.researchgate.net/publication/317182602_Pobreza_rural_ y_neoliberalismo_necesidades_de_las_familias_rurales_en_el_munic ipio_de_Ciudad_Barrios_Departamento_de_San_Miguel

10. Programa de las Naciones Unidas para el Desarrollo, (PNUD,2001). El desarrollo humano: pasado, presente y futuro. Informe sobre desarrollo humano. Pp. 11- 27.

11. Ríos M., Camacho E. (2016): "La agrobiodiversidad en várzea y su función económica en la Amazonía Peruana". http://www.redalyc.org/articulo.oa?id=357649703003

12. Stupino S., Iermanó M., Gargoloff, N., Bonicatto M. (2014): "La biodiversidad en los agroecosistemas". En: "Agroecología: bases 
teóricas para el diseño y manejo de agroecosistemas sustentables". Capítulo 5: 131-158.

13. http://sedici.unlp.edu.ar/handle/10915/37280

14. Sarandón S. (2009): "El agroecosistema: un ecosistema modificado" En: "Agroecología: bases teóricas para el diseño y manejo de agroecosistemas sustentables". Capítulo 4: 95-116.

15. http://sedici.unlp.edu.ar/handle/10915/37280

16. Vite M. (2007): "La Pobreza en Michoacán". http://www.redalyc.org/articulo.oa?id=40190107 